Para enlazar con este artículo / To link to this article:

http://dx.doi.org/10.6035/MonTI.2019.ne4.3

Para citar este artículo / To cite this article:

Conde Ruano, José Tomás. (2019) "El concepto de calidad en el doblaje para los estudiantes de traducción." En: Pérez L. de Heredia, María \& Irene Higes Andino (eds.) 2019. Multilingüismo y representación de las identidades en textos audiovisuales / Multilingualism and representation of identities in audiovisual texts. MonTI Special Issue 4, pp. 87-112.

\title{
EL CONCEPTO DE CALIDAD EN EL DOBLAJE PARA LOS ESTUDIANTES DE TRADUCCIÓN ${ }^{1}$
}

\author{
José Tomás Conde Ruano \\ tomas.conde@ehu.eus \\ Universidad del País Vasco (UPV/EHU)
}

\section{Resumen}

Este trabajo se basa en la opinión y los conocimientos de dos grupos de estudiantes de traducción en relación con el concepto de calidad en el doblaje. La información se ha recogido mediante cuestionarios electrónicos. Los estudiantes de traducción son conscientes de la importancia que tienen para la calidad tanto el ajuste (especialmente, la isocronía) como la interacción imagen-texto. Disponer del vídeo resulta vital para realizar una traducción completa y para evaluar la sincronización. Los estudiantes más avanzados demuestran una mayor seguridad y un mejor conocimiento de la actividad profesional. El perfil del estudiante de traducción resulta interesante para analizar el concepto de calidad en traducción audiovisual, si bien sería aconsejable contar con otros sujetos en el futuro.

\begin{abstract}
"The concept of quality in dubbing among translation students"

This paper deals with the opinion and beliefs of two groups of translation students on the concept of quality for dubbing. Data have been gathered by means of electronic questionnaires. Translation students are aware of the importance for quality of both synchronization (especially, isochrony) and the interaction of image and text. Video is essential to complete the translation task and to assess synchronies. Advanced students are more self-confident and show a wider knowledge of the professional activity. Translation students are an interesting profile to analyze the concept of quality in audiovisual translation, but further initiatives should consider other subjects as well.
\end{abstract}

1. Investigación realizada en el marco del proyecto IDENTITRA (FFI2012-39012-CO4-01T, MINECO). 
Palabras clave: Doblaje. Calidad. Evaluación. Pericia. Cuestionarios.

Keywords: Dubbing. Quality. Evaluation. Expertise. Questionnaires. 


\section{Introducción}

En los estudios sobre Traducción Audiovisual (en adelante, TAV) conviven diversos enfoques que tratan de explicar la actividad desde puntos de vista complementarios. Aunque esta modalidad vive un momento dulce, el concepto de calidad, clave en Traductología, no ha gozado de tanta consideración en la bibliografía específica sobre TAV.

El presente trabajo da cuenta de una investigación llevada a cabo con dos grupos de estudiantes de Traducción sobre algunas cuestiones relacionadas con el doblaje y la evaluación de su calidad. Por medio de cuestionarios, se pretende conocer la predisposición de este tipo de sujetos, así como cotejar los conocimientos alcanzados, que podrían reflejar diferencias debidas a la madurez o al nivel actual de pericia.

El estudio comienza con una breve exposición del problema; a continuación, se explica cómo se ha llevado a cabo el experimento para, después, exponer y discutir los resultados obtenidos, antes de presentar las conclusiones. Al final del trabajo, tras la bibliografía, se incluye una copia del cuestionario que ha servido de base para la investigación.

\section{Estado de la cuestión}

La investigación con estudiantes de traducción es habitual, quizás porque conforman un grupo accesible a los académicos que llevan a cabo los experimentos y dispuesto a colaborar sin poner trabas. Desde el punto de vista de los estudios inspirados en el concepto de pericia, son un grupo idóneo, ya que, por una parte, representan varios estadios intermedios entre los sujetos categorizados como "novatos" y aquellos que, tras años de experiencia, pasan a ser considerados "expertos". Así, en la nomenclatura de Hoffman (1998 apud Kiraly 2000: 58-59), serían sujetos "iniciados" (novatos que han iniciado una formación introductoria) o bien "aprendices" (estudiantes en formación más allá del nivel introductorio). Al poder ordenarlos en función del nivel de pericia alcanzado (esto es, del curso al que pertenecen), es posible comprobar a su vez qué conocimientos han pasado a formar parte de su acervo, qué actitudes han ido adquiriendo y cómo ha ido formándose su perfil como traductores 
en ciernes. Como resultado secundario, también es posible medir el éxito del proceso de enseñanza-aprendizaje o, lo que es lo mismo, ofrecer datos a los profesores de traducción sobre si se está logrando o no el desarrollo de las competencias necesarias para traducir.

Entre los estudios llevados a cabo con estudiantes, son habituales las iniciativas basadas en cuestionarios. Por un lado, los estudiantes están habituados a contestar encuestas y cuestionarios, lo cual hace que no sea necesario invertir tiempo alguno en formar a los sujetos en su utilización. Por otro lado, con un diseño adecuado, los cuestionarios permiten clasificar las respuestas y convertirlas en categorías mensurables, que posibilitan un análisis cuantitativo de los resultados y, en el mejor de los casos, su extrapolación. De hecho, la encuesta es la herramienta más utilizada en la investigación sociológica (Sierra Bravo 1994, apud Casas Anguita y otros 2003: 527) y resulta un método idóneo para recoger información relacionada con los gustos, hábitos y actitudes de los participantes.

Di Giovanni (2012: 496) afirma que "gathering and evaluating the opinions of receivers of translated films undoubtedly has great potential". En los últimos años, algunos estudios de recepción han incluido cuestionarios entre las herramientas de recogida de datos. Es el caso del trabajo de Ortiz-Boix y Matamala (2015: 19), en el que los destinatarios potenciales de una serie de documentales eran preguntados por sus gustos y preferencias. Lång y otros (2013: 77) también complementan su investigación basada en eye-trackers con un cuestionario; lo mismo hicieron Bourne y Lachat (2010:317) en su estudio sobre audiodescripción, Antonini (2005: 216) en su investigación sobre humor y subtitulado o De los Reyes (2015: 1-2) en su tesis sobre el doblaje. Son solo algunos ejemplos de cómo esta herramienta funciona para recabar datos en la investigación de la TAV, ya sea como método único, ya sea junto a otros.

Los conceptos de calidad y evaluación desde siempre han ocupado un espacio central en Traductología. Su presencia recurrente parece apuntar a una realidad huidiza, necesitada de investigaciones variadas, que usan métodos de todo tipo para iluminar áreas de una naturaleza resbaladiza y subjetiva. En la TAV son cada vez más comunes las iniciativas que, teniendo en cuenta las particularidades de la modalidad, abordan el concepto de la calidad y la actividad de la evaluación. Y es que son tan importantes en TAV que de ellos puede llegar a depender hasta el éxito (Díaz-Cintas 2003: 71) o la fama (Bittner 2011: 86) de una película.

Llegado a este punto, cabe preguntarse qué cuestiones específicas pueden estar relacionadas con la calidad en esta modalidad y no en otras. Para Mayoral (2005: 6) "la cuestión del ajuste o sincronización continúa siendo el eje central 
de todo estudio futuro sobre la traducción audiovisual dada su enorme repercusión sobre la calidad del producto final". Por su parte, Fuentes-Luque (2005: 139) considera que la sincronía es uno de los factores que afectan al éxito de la recepción de una producción audiovisual y De los Reyes (2015: 48) recoge la opinión de varios expertos que están de acuerdo en ver la sincronización visual como un factor determinante para la calidad del doblaje. La relevancia de la sincronía para el doblaje es subrayada tanto en general (Arumí et al. 2013: 47) como en relación con los distintos tipos de sincronías, sobre todo con los tres tipos que propone Chaume (2012: 68-69):

- sincronía labial o fonética: la que ajusta el movimiento de los labios con el sonido que se escucha;

- sincronía cinésica: la que ajusta el movimiento del cuerpo con lo que se dice;

- isocronía: la que ajusta el tiempo de los sonidos y las pausas de los actores.

Entre los tres tipos, el propio Chaume (2004: 279 y 2012: 68) considera que la falta de isocronía es probablemente el error más penalizado por la audiencia y, por ello, el que más íntimamente está ligado al concepto de calidad.

Otro asunto que suele aparecer con frecuencia en la bibliografía sobre TAV es el papel crucial que desempeña la imagen para traducir, ya que, en esta modalidad de traducción, a diferencia de otras, interactúan los códigos lingüístico e iconográfico (Chaume 2004: 232; Chaume 2012: 110-112; Segovia 2005: 85). Algunos autores critican que, en ocasiones, el traductor audiovisual tenga que trabajar sin ver el vídeo del producto audiovisual, lo cual afecta sin duda a la calidad de su trabajo: para Chaume (2004: 268-269) y Sokoli (2005: 185), traducir sin apoyo visual puede llevar al traductor a pasar por alto algunas cuestiones como por ejemplo algunas relacionadas con la sincronía cinésica. Si no disponer de la imagen puede afectar a la calidad de lo que se traduce, cabe pensar que igualmente afecta a lo que se evalúa.

¿Y sobre quién recae la responsabilidad de evaluar el doblaje? Díaz-Cintas (2003: 106) habla de la figura del supervisor de traducción, que es quien debe velar por la calidad del producto. Para Abdallah (2012: 36), resulta difícil aunar criterios de calidad en un servicio en el que participan tantos actores diferentes. Por su parte, Alfaro de Carvalho (2012: 465) señala a los propios traductores, esto es, alude en cierto modo a la autoevaluación. Hay otros trabajos en los que la evaluación de productos audiovisuales se aborda desde el punto de vista de la investigación. Es el caso de Nord y otros (2015: 8) quienes incluyen a los propios investigadores como evaluadores de las traducciones audiovisuales; en 
cambio, en el experimento de Ortiz-Boix y Matamala (2015: 19) participaron tres grupos diferentes de evaluadores: expertos (un grupo de profesores de TAV especializados en voces superpuestas), un estudio de doblaje (actores, director de doblaje y técnico) y usuarios finales. De hecho, la mayoría de autores subrayan el papel del receptor como evaluador de la calidad (entre otros, Abdallah 2012: 36; Alfaro de Carvalho 2012: 470 y Zabalbeascoa 2008: 172).

Mientras no surjan más estudios en los que se mida la recepción de la audiencia o se interprete qué entiende el espectador por una TAV de calidad, del tipo de los que proponen estudiosos como Chaume (2007: 78) o Di Giovanni (2012: 496), puede resultar interesante conocer de primera mano qué opinión tienen los estudiantes sobre las cuestiones de calidad que afectan a la TAV, a la vez que se les cuestiona sobre otros temas polémicos en la bibliografía sobre el doblaje. No en vano, Gambier (2009 apud De los Reyes 2015: 56) sugería indagar en la recepción a través de cuestionarios y entrevistas, entre otros métodos.

\section{Materiales y métodos}

Se diseñó un cuestionario con el que se pretendía conocer cuál es el concepto de calidad en el doblaje y en la TAV en general para los estudiantes de traducción. La administración del cuestionario es solo una parte de una prueba más amplia sobre la evaluación del doblaje; sin embargo, el presente trabajo se centra en los resultados del cuestionario. ${ }^{2}$

Se contó con estudiantes del Grado en Traducción e Interpretación de la Universidad del País Vasco / Euskal Herriko Unibertsitatea (UPV/EHU). En concreto, se partió de dos grupos de alumnos: el primero lo integraban estudiantes de segundo curso (iniciados); el segundo, estudiantes de cuarto (aprendices). Para acceder a ellos fue necesaria la colaboración de las profesoras de dos asignaturas de traducción: Prácticas de traducción I: inglés-español I, ofertada en segundo y Prácticas de traducción V: inglés-español y españolinglés III, ofertada en cuarto. Las asignaturas guardaban varias similitudes: eran impartidas al alimón por las mismas profesoras, se basaban en la misma combinación lingüística (inglés-español), equivalían al mismo número de ECTS (6) y se desarrollaban en el mismo cuatrimestre (el primero). Por todas estas razones, se podía esperar que los estudiantes de las asignaturas fueran

2. La prueba comenzaba con una tarea de evaluación del doblaje, para la que los estudiantes partían de la escena en inglés y en español, bien como vídeos, bien como transcripciones de las mismas. Los resultados de la prueba de evaluación han sido ya analizados y se encuentran en proceso de publicación (Conde Ruano, de próxima aparición). La segunda parte de la investigación se basaba en el cuestionario, cuyos resultados se comentan en el presente trabajo. 
comparables ya que, a priori, lo único que los diferenciaba era el grado de pericia alcanzado en traducción.

Una vez que se contó con la colaboración de las profesoras, el siguiente paso era decidir de qué manera se iba a medir el conocimiento de los informantes sobre el concepto de calidad en el doblaje, así como su predisposición. Como se iba a llevar a cabo también un experimento en clase, se decidió utilizar un cuestionario que sería administrado tras la primera prueba de evaluación. Para su diseño se tuvo en cuenta que su cumplimentación no llevara a los sujetos demasiado tiempo. Con ello se buscaba una implicación mayor de los estudiantes que podrían haberse visto desmotivados si se hubieran encontrado un ejercicio demasiado extenso. Se buscó conjugar preguntas que, por un lado, ayudaran a perfilar a los sujetos participantes (para hallar posibles diferencias y coincidencias entre los estudiantes de ambos cursos) y, por otro, sirvieran para recabar información sobre lo que opinan acerca de diversas cuestiones relacionadas con los conceptos de calidad y evaluación en el doblaje. Para elegir las preguntas concretas se partió de un análisis previo de la bibliografía afín, comentada en la sección 2 de este trabajo.

Se apostó por un cuestionario en formato digital, para agilizar la recogida de datos y su posterior análisis. Se utilizó asimismo un formato sencillo (una tabla en MS Word), buscando una vez más que el ejercicio resultara fácil, familiar y cómodo para los informantes. Cuando el diseño se hubo completado, se subió a la plataforma de Consigna de la UPV/EHU protegido por una contraseña.

Los dos grupos de estudiantes se enfrentaron a la prueba previa y al cuestionario la misma mañana; se buscaba evitar el cruce de informaciones entre ambos grupos, o, lo que es lo mismo, que todos los estudiantes contestaran a la encuesta con el mismo grado de frescura. Los alumnos de cada grupo tuvieron que seguir las instrucciones, que consistían en lo siguiente:

- descargar los archivos de la plataforma de Consigna;

- realizar la prueba de evaluación;

- contestar al cuestionario;

- enviar los resultados por correo electrónico.

La prueba era anónima. No obstante, para que pudieran compararse los resultados de la prueba de evaluación con los de las encuestas, a cada estudiante se le facilitó un código al azar, que tenía que escribir tanto en la hoja de evaluación como en el cuestionario. En la sesión, que no duró más de 45 minutos entre una prueba y la otra, el investigador estaba disponible para resolver las dudas 
que pudieran surgir. No obstante, las dudas fueron escasas durante las dos sesiones (con los estudiantes iniciados y con los aprendices).

Como se querían utilizar dos grupos comparables, se tuvo en cuenta los cuestionarios enviados por 16 estudiantes de cada curso. Algunos estudiantes más estaban presentes en clase y realizaron el cuestionario, pero el análisis se basó en los cuestionarios de un total de 32 sujetos. La manera de elegir 16 de cada grupo se basó en el sistema siguiente: en segundo, había 16 códigos encabezados por la cifra 2 y en cuarto, 16 encabezados por la cifra 4. Además, se prepararon otros códigos encabezados bien por la cifra 1, bien por la 3. En la clase de segundo había 18 alumnos presentes, con lo que se utilizaron los 16 códigos encabezados por el 2 y dos encabezados por el 1 . Se mezclaron y repartieron al azar, de manera que para el análisis se descartaron las dos encuestas que comenzaban por el 1 . En la clase de cuarto intervinieron 24 alumnos y fueron ocho las encuestas descartadas: todas las que recibieron un código encabezado por un 3 en vez de por un 4. La única manipulación que se introdujo en el reparto de los códigos se produjo cuando había algún estudiante Erasmus presente, a quienes directamente se les daba un código que comenzara por 1 o por 3; con ello, se pretendía que el perfil de los sujetos analizados fuera lo más parecido posible, en el sentido de que se evitara que variables extrañas (como la lengua materna o el país de origen) distorsionaran los datos.

Cuando se hubo comprobado que los alumnos habían enviado correctamente el cuestionario a la dirección de correo facilitada, las profesoras retomaron sendas clases.

Para el análisis de los datos, se copió y pegó toda la información en una hoja de cálculo de Microsoft Excel. La mayoría de operaciones se realizaron en este programa; se contempló la posibilidad de analizar los datos con programas de tratamiento estadístico, pero, al partir de un número pequeño de informantes y de preguntas abiertas, se concluyó que resultaría más operativo trabajar directamente sobre la hoja de cálculo. El análisis se basó en las siguientes hipótesis:

1) La mayoría de estudiantes estarían predispuestos positivamente a la traducción audiovisual.

2) La mayoría de sujetos creería imprescindible el uso de la imagen para la traducción audiovisual y para su evaluación.

3) De entre los parámetros de calidad mencionados para el doblaje, la sincronización ocuparía un lugar cardinal (y, entre los tipos de ajuste, destacaría el papel de la isocronía).

4) Los alumnos de cuarto o aprendices mostrarían una mayor seguridad al responder que los de segundo o iniciados, debido a su conocimiento más avanzado tanto de sus gustos como de la profesión. 


\section{Resultados y discusión}

El análisis de los resultados se divide en dos partes: en primer lugar, se abordan las preguntas relacionadas con el perfil de los que han participado en el estudio; a continuación, se comentan las respuestas relacionadas específicamente con la actividad de la TAV, con su calidad y su evaluación.

\subsection{Perfil personal}

Para presentar el análisis, se ha dividido la información en dos campos: la que identifica al sujeto y la que resume sus hábitos y gustos.

\subsubsection{Identificación}

La pregunta más evidente era aquella con que se pretendía conocer el curso en el que estaban matriculados los estudiantes. Así y todo, uno de los sujetos del grupo de $2^{\circ}$ señaló cursar $3^{\circ}$, lo cual podría deberse a que el año anterior hubiera estado de intercambio o no hubiera aprobado la asignatura. El resto de iniciados dijeron estar matriculados en $2^{\circ} \mathrm{y}$ todos los aprendices respondieron también que en $4^{\circ}$.

$\mathrm{Al}$ tratarse de estudiantes pertenecientes a dos cursos distintos, lo normal es que se hallen diferencias en la edad media de ambos grupos. La tabla 1 muestra la edad media de los informantes, así como el mínimo, el máximo y el rango entre los sujetos de mayor y menor edad en cada grupo.

Tabla 1. Edad

\begin{tabular}{|l|l|l|}
\hline & \multicolumn{1}{|c|}{ Segundo } & \multicolumn{1}{c|}{ Cuarto } \\
\hline Mínimo & 19.00 & 21.00 \\
\hline Máximo & 40.00 & 22.00 \\
\hline Rango & 21.00 & 1.00 \\
\hline Media & 21.63 & 21.19 \\
\hline
\end{tabular}

Si se atiende solo a la media, puede sorprender que los iniciados son mayores que los aprendices. No obstante, si se tiene en cuenta el rango, se descubre cómo los de $4^{\circ}$ son un grupo más homogéneo que los de $2^{\circ}$, entre los que se encuentra un sujeto con 40 años y otros dos con 23 y 24 años, respectivamente, que hacen subir la media del subgrupo.

El gráfico 1 presenta los resultados relativos a la lengua materna de los sujetos. 


\section{Gráfico 1. Lengua materna}

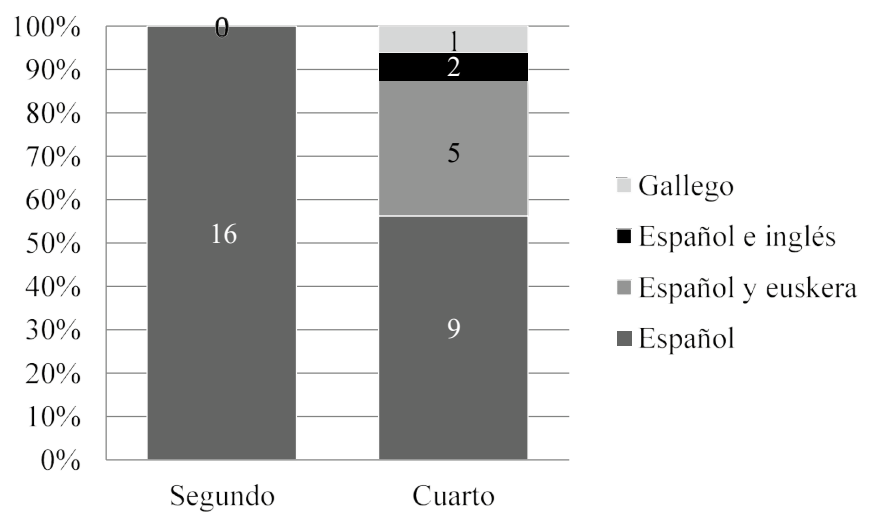

Aparece la misma homogeneidad, aunque en este caso son los iniciados quienes muestran un perfil más coincidente. Entre los aprendices hay nativos de español, bilingües con euskera o inglés y hasta un nativo de gallego. Esta disparidad entre los dos grupos resulta chocante e inesperada y podría deberse, quizás, a un error de interpretación del cuestionario: es posible que los estudiantes de $2^{\circ}$ creyeran que solo debían mencionar una lengua, mientras que los de $4^{\circ}$ quizás entendieron que podían señalar más de una.

La última pregunta de este bloque versa sobre el nivel de inglés de los sujetos, ya que participaban en una asignatura de prácticas inglés-español. El gráfico 2 muestra los resultados, divididos por subgrupos.

Gráfico 2. Nivel de inglés

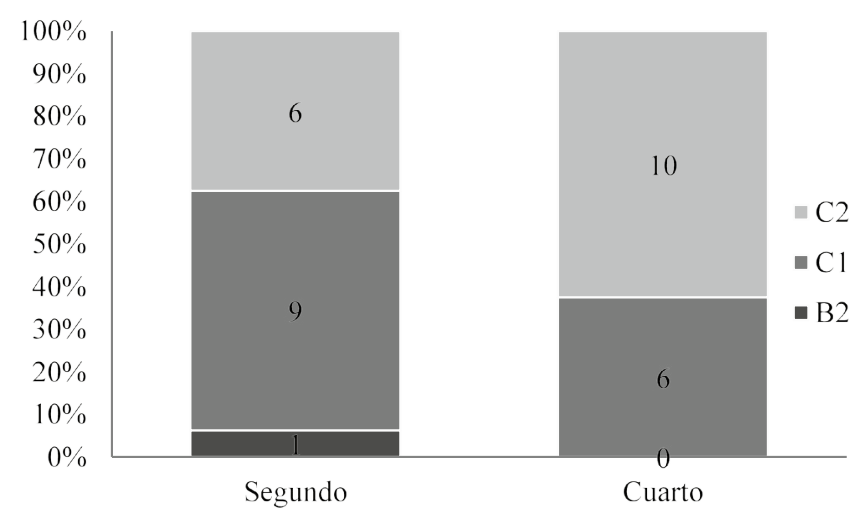


La mayoría de sujetos salvo 1 (en el grupo de los iniciados) habían acreditado al menos el nivel $\mathrm{Cl}$ de inglés. Figuran más sujetos con el nivel $\mathrm{C} 2$ en cuarto, quizás porque en esos dos años de diferencia entre ambos cursos algunos alumnos aprovechan para obtener la máxima certificación de inglés.

En resumen, los dos subgrupos de estudiantes que participaron en el estudio presentaban un perfil parecido, típico al que se les presupone como estudiantes de traducción en $2^{\circ}$ y $4^{\circ}$ curso, respectivamente. El único dato que resulta sorprendente, dado el contexto en el que se desarrolló el cuestionario, es que ninguno de los estudiantes iniciados se presentara a sí mismo como bilingüe de español y euskera.

\subsubsection{Hábitos y gustos}

Se les preguntó también por sus hábitos. Por una parte, se pretendía descubrir si están acostumbrados a ver películas y series dobladas y, por otra, si están familiarizados con la lectura de guiones. El gráfico 3 muestra las respuestas a ambas preguntas.

Gráfico 3. Costumbre de ver productos doblados y de leer guiones

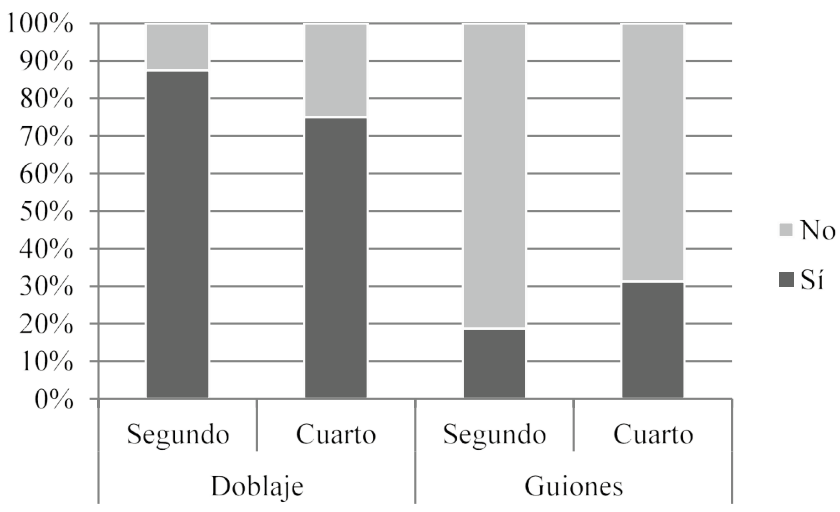

Con independencia del subgrupo al que pertenecen, la mayoría de sujetos dicen estar habituados a ver películas y series dobladas, pero no a leer guiones o transcripciones de ese tipo de obras. Las diferencias son escasas entre los dos subgrupos, si bien el hábito del doblaje está más extendido entre los iniciados y el de la lectura de transcripciones, entre los aprendices. Esto último puede deberse a que los alumnos de $4^{\circ}$ han empezado a trabajar la TAV recientemente 
en clase y, así, los que respondieron afirmativamente, pudieron haberse referido a la experiencia atesorada en el entorno académico.

En general, hay que decir que varios estudiantes afirmaron estar familiarizados con el doblaje en el cine pero que, cuando ven series en casa, prefieren hacerlo en versión original. La preferencia por la versión original en estudiantes de traducción es irónica pues, si bien es importante que el traductor en ciernes aproveche el material audiovisual para poner en práctica sus destrezas lingüísticas, alejarse del material doblado no parece la mejor manera de acostumbrarse a las prácticas utilizadas por los profesionales del sector, a las convenciones del doblaje que, en algunos casos, tendrán que interiorizar si van a dedicarse a la TAV como actividad principal.

En cualquier caso, se les preguntó específicamente por su vocación como traductores audiovisuales en particular y como traductores en general. Las respuestas dadas por los informantes fueron a veces poco contundentes: por ello, para las respuestas que no fueron un "Sí" o un "No" rotundo, se ha introducido la categoría "Matiz". Ejemplos de respuestas categorizadas como "Matiz" serían, entre otras, las siguientes: "No estoy seguro, pero creo que no" (sujeto 403), "Yo creo que sî" (212), "Puede" (204) o "Me llama muchísimo la atención, pero hasta que no lo experimente no lo sabré con seguridad" (201). El gráfico 4 presenta los datos divididos por subgrupos.

Gráfico 4. Vocación por la traducción y por la TAV

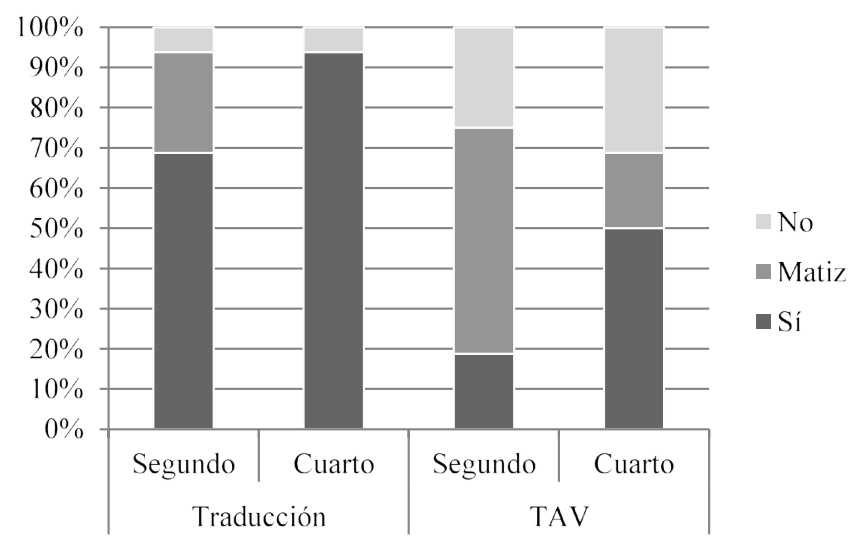

Comparados con los de $4^{\circ}$, los alumnos de $2^{\circ}$ presentan más a menudo respuestas ambiguas o cautas tanto a una pregunta como a la otra. El resultado es lógico: una de las funciones de la formación es que los discentes devengan 
conscientes de si les gusta o no aquello que están estudiando. En ese sentido, los aprendices están mucho más seguros que los iniciados tanto sobre su vocación como traductores (casi unánime) como por la TAV (al 50 \%). Tampoco es de extrañar que haya menos dudas en torno a la traducción en general, pues no todos los estudiantes tienen por qué querer dedicarse a la misma modalidad de traducción. En cualquier caso, la cantidad de alumnos que dicen sentir vocación por la TAV no es nada desdeñable y tampoco ha de extrañar en una época en la que los medios audiovisuales gozan de una posición privilegiada entre las opciones de ocio de los jóvenes.

La última pregunta que sirve para perfilar a los informantes de esta investigación es la que inquiría sobre la experiencia atesorada en TAV. Aunque era una pregunta directa, que se podía responder con un "Sí" o un "No", la mayoría de sujetos matizaron la respuesta. Así, además de las dos categorías binarias, se han tenido en cuenta dos categorías más: "Clase", para aquellos que decían tener experiencia en TAV porque la habían obtenido en clase, esto es, en las asignaturas prácticas de traducción en la Universidad; y "Afición", para aquellos que dijeron haber practicado la TAV, pero no de manera profesional, sino subtitulando vídeos de YouTube y, en general, como entretenimiento. El gráfico 5 presenta los resultados obtenidos en cada grupo.

\section{Gráfico 5. Experiencia en TAV}

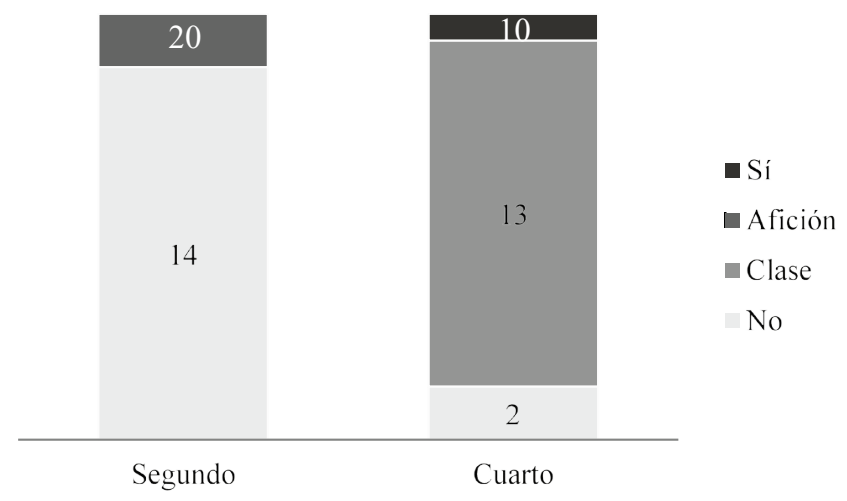

Como cabía esperar, los alumnos de $2^{\circ}$ no tienen experiencia en TAV, aunque dos de ellos dicen haberla practicado como entretenimiento. La respuesta más habitual entre los alumnos de $4^{\circ}$ es la categorizada como "Clase": salvo en tres casos (un alumno dice tener experiencia y dos responden negativamente), no 
se atreven a decir de forma tajante que tienen experiencia en TAV, aunque, por otra parte, quieren dejar patente que han practicado esta modalidad de traducción y, por ello, tampoco han respondido "No".

\subsection{TAV y calidad}

Una vez trazado el perfil medio de los sujetos que respondieron al cuestionario, resta comentar en profundidad qué es lo que ambos subgrupos conocen y opinan del oficio del traductor audiovisual, sobre todo en relación con los conceptos de calidad y evaluación.

\subsubsection{Ejercicio de la TAV}

El cuestionario incluía dos preguntas relacionadas con dos de los problemas acuciantes para la TAV. La primera pregunta se refiere a la necesidad de contar con el vídeo de la obra audiovisual en el momento de realizar la traducción. Todos los sujetos dijeron que era imprescindible, salvo dos iniciados, que, si bien no dijeron que lo fuera, al justificar su respuesta señalaron que era "recomendable" contar con el vídeo para traducir.

Más que el porcentaje en sí, que, como puede apreciarse, rondó el $100 \%$ de respuestas afirmativas, llaman la atención las justificaciones de los sujetos a sus respuestas, pues fueron de lo más variopinto. Algunos se refirieron a la interacción entre la imagen y el texto: el ajuste labial, la isocronía o la sincronía cinésica. Un ejemplo sería el sujeto 202, que dice "Creo que resulta fundamental para que luego el ajuste sea correcto y el producto quede natural”. Los demás sujetos aludieron a la comprensión del original, a saber: la importancia del contexto y la escenografía para interpretar bien los matices de la obra. En este sentido, puede citarse como ejemplo al sujeto 416, que responde: "Sí, porque los elementos paralingüísticos también entran en juego y pueden modificar la traducción". El gráfico 6 muestra las respuestas agrupadas y divididas por subgrupo de estudiantes. Es preciso señalar que no todos los sujetos arguyeron razones y que algunos mencionaron más de una justificación, por lo que los números no reflejan la cantidad de sujetos, sino la cantidad de menciones realizadas sobre cada tipo. 
Gráfico 6. Importancia de la imagen para traducir obras audiovisuales

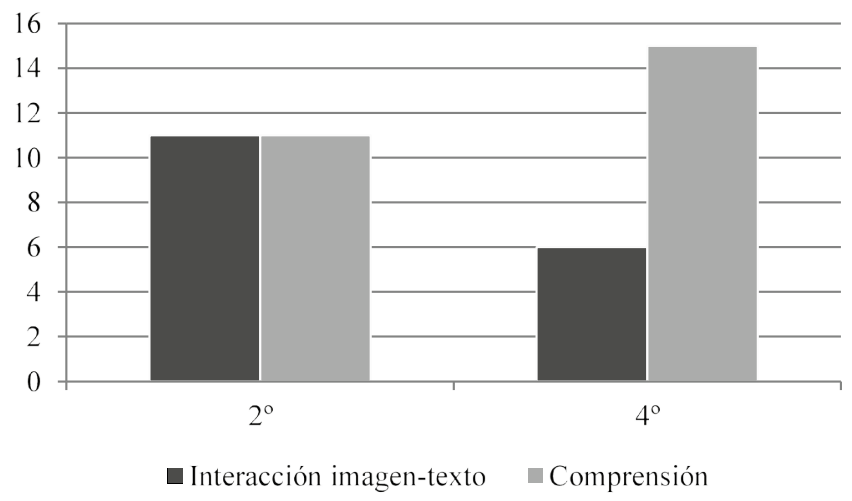

Mientras que los alumnos de $2^{\circ}$ aportan justificaciones de los dos tipos al mismo nivel, los de $4^{\circ}$, que también señalan razones de ambos tipos, destacan en señalar la importancia de contar con la imagen para comprender el texto original, esto es, para no dejarse nada en el proceso de traducción. En consecuencia, cuando un traductor iniciado piensa en TAV lo que antes le viene a la cabeza es la importancia de la sincronización. En cambio, aquellos que ya se han iniciado en esta práctica consideran que la imagen es imprescindible, no solo para realizar una buena sincronización, sino también y sobre todo para lograr una traducción completa de la obra audiovisual. Es decir, ambos grupos de estudiantes conocen la relevancia de la conexión imagen-texto en TAV, pero, mientras que los de $2^{\circ}$ se refieren más a la dificultad técnica, los de $4^{\circ}$ demuestran haber aprendido que esa interacción no se queda en la superficie de lo técnico, sino que es más profunda y afecta a la misma hermenéutica del producto audiovisual.

Otra de las cuestiones problemáticas y ampliamente tratadas en la bibliografía sobre el doblaje es quién debería ser el encargado de ajustar el texto, si el propio traductor $\mathrm{u}$ otra persona. Al respecto, se incluyó una pregunta en el cuestionario y las respuestas fueron diferentes entre los dos subgrupos, como muestra el gráfico 7 . 
Gráfico 7. Traducción y ajuste por la misma persona

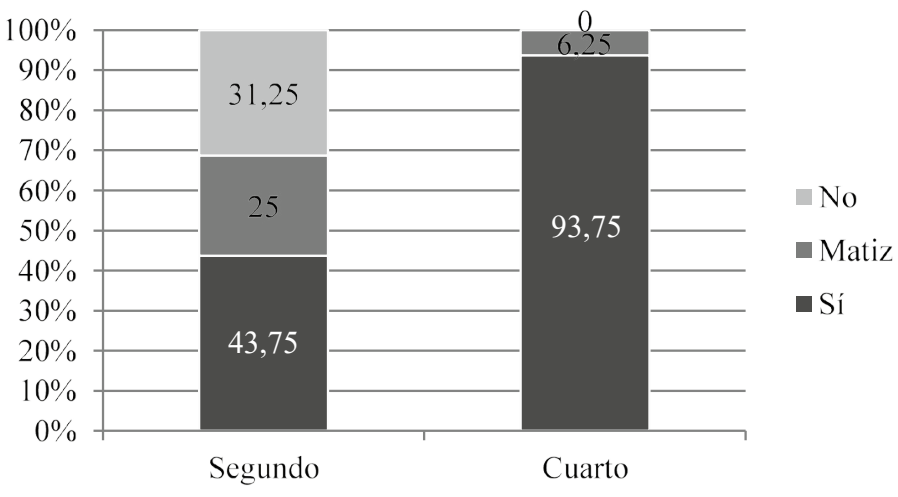

Los alumnos de $2^{\circ}$ no tienen una opinión formada sobre el tema, como muestra la disparidad de respuestas que han dado. Por el contrario, los de $4^{\circ}$ son casi unánimes al responder que sí, que el propio traductor es quien debería hacerse cargo del ajuste. Es presumible que este resultado se deba a la función docente, ya que los alumnos de $4^{\circ}$ han empezado a familiarizarse con la problemática de la TAV.

Entre aquellos que han respondido "no", hay justificaciones ambiguas (algunos sujetos sugieren que debe hacerlo "una persona"). Las razones por las que se considera que el traductor debe encargarse de esta función muestran también el desconocimiento patente de los alumnos de $2^{\circ}$ pues solo uno de los 16 (el sujeto 205) aportó razones: "saben el tiempo que ocupan los subtítulos en relación con el audio"; por su parte, los de $4^{\circ}$ fueron algo más profusos al respecto: un sujeto afirmó que el ajuste debe hacerlo el traductor porque está formado para ello; dos, porque es el único que conoce los dos idiomas y otros cuatro sujetos basaron su opinión en la idea de que el traductor es la persona que mejor conoce la tarea y el documento, por lo que es también la que está en mejor disposición de realizar el ajuste.

\subsubsection{Calidad y evaluación}

El resto de preguntas del cuestionario versaban sobre la evaluación de productos audiovisuales y sobre el concepto de calidad en TAV. La primera pregunta es similar a aquella cuyos resultados quedaron ilustrados por el gráfico 6 pero, en este caso, se pretendía conocer la opinión de los estudiantes sobre si era necesario contar con el vídeo a la hora de evaluar la calidad de la TAV. La tabla 2 muestra el porcentaje de cada tipo de respuesta: 
Tabla 2. Importancia de la imagen para evaluar productos audiovisuales

\begin{tabular}{|c|c|c|c|}
\hline & Sí & Matiz & No \\
\hline Segundo & 62.5 & 18.75 & 18.75 \\
\hline Cuarto & 81.25 & 18.75 & 0 \\
\hline
\end{tabular}

Las respuestas categorizadas como "Matiz" se refieren, por ejemplo, a aquellas en las que los sujetos decían que no era imprescindible pero sí recomendable contar con el vídeo (el sujeto 201, por ejemplo, dice "aunque no tan importante como en el caso de traducirlo"). Los datos muestran que es bastante relevante para los alumnos que el evaluador del producto audiovisual cuente con la imagen, aunque las respuestas no son tan unánimes como en el caso de aquellas que respondían a la pregunta sobre si era imprescindible el vídeo para realizar la TAV. El gráfico 8 muestra los datos agrupados en las dos categorías principales.

Gráfico 8. Importancia de la imagen para evaluar productos audiovisuales (justificaciones)

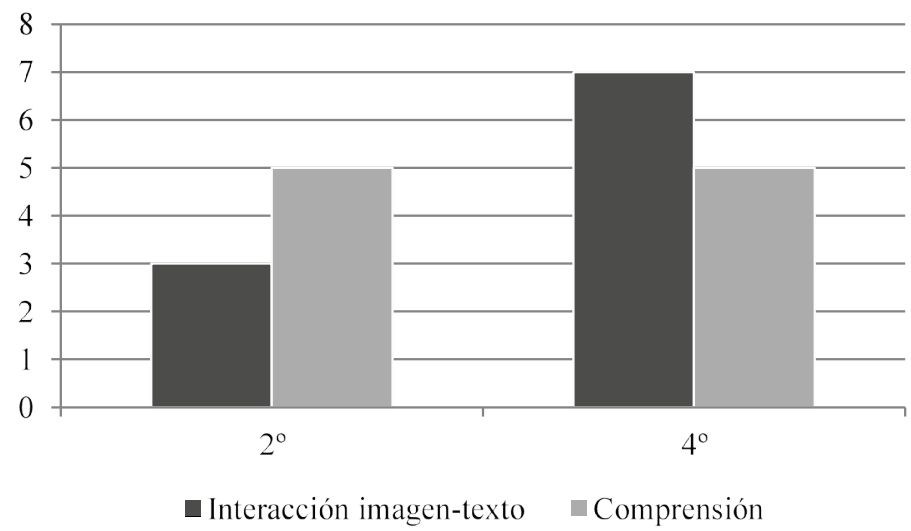

En esta ocasión, las diferencias son más notables en el caso de las respuestas relacionadas con la interacción imagen-texto, más citadas por los estudiantes de $4^{\circ}$ que por los de $2^{\circ}$. Comparando con el gráfico 6 , parece que los estudiantes son conscientes de la importancia de la imagen para las actividades relacionadas con la TAV pero con un matiz: a la hora de traducir, contar con la imagen es crucial sobre todo para comprender bien el texto y para reflejar en el producto traducido todo lo que estaba en el original; a la hora de evaluar el texto, en cambio, la imagen es importante sobre todo para comprobar que el ajuste se ha realizado correctamente. Así, la interacción imagen-texto y la comprensión 
son dos aspectos relevantes en la percepción de la calidad de los productos audiovisuales, aunque cada uno de ellos desempeña un papel diferente según el proceso en el que se esté, el de traducción o el de evaluación.

También se preguntó a los informantes quién debía evaluar las traducciones audiovisuales. Las respuestas aparecen en la tabla 3, ordenadas de mayor a menor según el número de menciones totales

Tabla 3. Sujeto evaluador

\begin{tabular}{|l|c|c|c|}
\hline & $\mathbf{2}^{\mathbf{0}}$ & $\mathbf{4}^{\mathbf{0}}$ & Total \\
\hline Traductor & 5 & 5 & 10 \\
\hline Espectador, audiencia & 4 & 4 & 8 \\
\hline Bilingüe o especialista en lenguas & 0 & 8 & 8 \\
\hline Otro traductor & 3 & 4 & 7 \\
\hline Doblador & 4 & 1 & 5 \\
\hline Corrector & 3 & 1 & 4 \\
\hline Director o director de doblaje & 2 & 2 & 4 \\
\hline Todo el equipo o equipo técnico & 2 & 1 & 3 \\
\hline NS/NC & 1 & 0 & 1 \\
\hline Crítico & 1 & 0 & 1 \\
\hline Guionista & 1 & 0 & 1 \\
\hline Productor & 1 & 0 & 1 \\
\hline Experto en cine & 0 & 1 & 1 \\
\hline Experto en traducción & 0 & 1 & 1 \\
\hline
\end{tabular}

Dejando aparte las respuestas anecdóticas, la que más se repite es la de "Traductor", entendiéndose como la misma persona que ha realizado la traducción. Del mismo modo, el "espectador" es citado en los dos subgrupos con bastante frecuencia, así como "otro traductor" y, en menor medida, el "director". Las diferencias más significativas se hallan en la categoría "bilingüe o especialista en lenguas" que solo mencionan los aprendices y, además, es en ese subgrupo la respuesta más habitual. La variable lingüística es tenida en cuenta por los estudiantes avanzados para los que el conocimiento de lengua extranjera se destaca como un mínimo que debe tener quien quiera evaluar una traducción, mientras que los de $2^{\circ}$, más inexpertos, creen que la evaluación del trabajo la han de hacer casi siempre los mismos que han desarrollado ese trabajo, excepción hecha del público, que también juega un papel importante. 
Es decir, los alumnos de $2^{\circ}$ abogan más por la autoevaluación (ya sea individual, ya sea en grupo) y los de $4^{\circ}$ citan más a menudo a personas en principio ajenas del proceso de traducción, quizás porque están más acostumbrados a que su trabajo sea evaluado y conocen las bondades de la evaluación por parte de terceros.

Las dos últimas preguntas son acerca del concepto de calidad en TAV. En la primera, los sujetos tenían que ordenar de 1 (menos) a 3 (más) los tres tipos de discronías en función de su gravedad. Se utilizaron descripciones fáciles de entender. Así, se ha categorizado como asincronía "labial" lo que en la encuesta aparece como "no coincidan con el movimiento de los labios de los actores"; falta de "isocronía", "que dure bastante más o menos que el parlamento de los actores" y asincronía "cinésica", "que no se tenga en cuenta los movimientos corporales de los actores". Para facilitar el análisis se ha otorgado 1 punto al tipo de problema que los sujetos consideran menos grave para el doblaje; 3 puntos, para el que más y 2 , para el otro. La tabla 4 muestra el valor medio recibido por cada problema en cada subgrupo, así como la moda o valor más repetido.

Tabla 4. Importancia de los tipos de discronías

\begin{tabular}{|l|c|c|c|c|}
\hline & \multicolumn{2}{|c|}{$2^{\text {o }}$} & \multicolumn{2}{c|}{$4^{\text {o }}$} \\
\hline & Media & Moda & Media & Moda \\
\hline Labial & 2.07 & 2 & 1.80 & 2 \\
\hline Isocronía & 2.47 & 3 & 2.53 & 3 \\
\hline Cinésica & 1.47 & 1 & 1.67 & 2 \\
\hline
\end{tabular}

Tanto un grupo como el otro consideran, a la vista de los resultados, que el problema de ajuste más grave para el doblaje es la falta de isocronía; además, los dos grupos coinciden en señalar la falta de isocronía cinésica como el problema menos importante de los tres. La única diferencia entre los dos grupos es que la falta de sincronía cinésica recibe casi siempre un punto entre los estudiantes iniciados y 2 entre los aprendices, aunque las diferencias, teniendo en cuenta la media, no pueden considerarse significativas.

Por último, se incluía una pregunta sobre cuáles son los criterios que hay que utilizar para valorar la calidad de los productos audiovisuales. Al ser una pregunta abierta, se han categorizado las respuestas, que se presentan en la tabla 5. 
Tabla 5. Criterios de calidad en los productos audiovisuales

\begin{tabular}{|l|c|c|c|}
\hline & $\mathbf{2}^{\mathbf{0}}$ & $\mathbf{4}^{\mathbf{0}}$ & Total \\
\hline Ajuste correcto & 10 & 12 & 22 \\
\hline Naturalidad, adaptada a la lengua de llegada & 7 & 6 & 13 \\
\hline Fidelidad al contenido & 8 & 5 & 13 \\
\hline Conserva el tono o el registro, oralidad, realismo & 4 & 6 & 10 \\
\hline Tiene en cuenta elementos no lingüísticos & 3 & 3 & 6 \\
\hline La traducción en sí & 3 & 3 & 6 \\
\hline Los elementos culturales & 1 & 4 & 5 \\
\hline Misma función o finalidad & 2 & 1 & 3 \\
\hline Respetar las convenciones técnicas, segmentación & 0 & 3 & 3 \\
\hline Los mismos que para una obra de teatro & 1 & 0 & 1 \\
\hline Precisión del lenguaje & 0 & 1 & 1 \\
\hline Elecciones léxicas & 0 & 1 & 1 \\
\hline Coherencia & 0 & 1 & 1 \\
\hline Modulación y expresividad de la voz & 0 & 1 & 1 \\
\hline
\end{tabular}

Las razones relacionadas con el ajuste son las más mencionadas en los dos subgrupos de sujetos, lo cual demuestra el valor de este factor para la evaluación de la calidad en TAV. En general, los alumnos de $4^{\circ}$ citan un mayor número de criterios de calidad y algunos de ellos, como la modulación y expresividad de la voz o la segmentación, apuntan a un mayor conocimiento de la modalidad en sí que sus colegas de $2^{\circ}$ curso. Por lo demás, los números son parejos, aunque la diferencia más reseñable es, tal vez, la buena opinión que tienen los iniciados de la fidelidad al contenido o al mensaje, mientras que entre los aprendices hay al menos tres criterios más destacados: el ajuste (ya comentado), la naturalidad y la oralidad.

No es descabellado afirmar que lo que los alumnos dicen sobre la calidad de la TAV ofrece pistas sobre lo que dichos alumnos conocen sobre la TAV; de aquí también la mayor alusión a los culturemas entre los alumnos de $4^{\circ}$ : probablemente por la experiencia obtenida en clase saben ya que ese es uno de los problemas más habituales en el doblaje, mientras que los alumnos de $2^{\circ}$ se han referido a problemas más generales, comunes a otros tipos de modalidades, excepción hecha de la sincronización, que es quizás el elemento distintivo de la TAV, tanto para aprendices como para iniciados. 


\section{Conclusiones}

Este trabajo supone un primer acercamiento a lo que piensan los estudiantes de traducción sobre el concepto de calidad en la TAV y en la modalidad del doblaje en particular.

Los estudiantes de traducción resultan un grupo idóneo para investigar distintas cuestiones relacionadas con el concepto de pericia, ya que representan distintos estadios de aprendizaje y, por ello, es fácil apreciar en ellos cómo se van interiorizando conceptos y conocimientos. Además, responden bien a los trabajos basados en cuestionarios, pues están más que habituados a cumplimentarlos. Estudiar la calidad en TAV implica poner énfasis en aquellos aspectos que hacen de esta modalidad una actividad diferente: por ello, un estudio sobre lo que opinan los estudiantes de traducción acerca de este concepto debe tener en cuenta puntos controvertidos y habituales en la bibliografía consultada.

La sincronización es quizás el aspecto más destacado en los estudios sobre doblaje, y así ha quedado reflejado en las respuestas de los alumnos, quienes además han confirmado la segunda hipótesis: la falta de isocronía es, para ellos, el error de ajuste más grave. Esta opinión no puede deberse únicamente al consejo de los profesores pues los estudiantes de $2^{\circ}$, con un conocimiento más intuitivo y formación todavía nula en TAV, opinan exactamente lo mismo que los de $4^{\circ}$. En lo que sí difieren es en conocimientos de tipo más profesional, ligados también a la sincronía: los aprendices, por ejemplo, tienen claro que lo ideal es que ajuste y traducción lo haga la misma persona, mientras que las respuestas de los iniciados son más variadas. En general, esta falta de seguridad en las respuestas de los estudiantes de $2^{\circ}$ ha sido la tendencia a lo largo de todo el análisis, lo que confirma a su vez la hipótesis cuarta expuesta en la sección 3.

La tercera hipótesis también ha sido confirmada, pues ha quedado patente la importancia de la imagen cuando se trabaja con productos audiovisuales para los estudiantes de traducción. El resultado más interesante al respecto es la diferencia que se deja traslucir en el porqué de esa relevancia: que los estudiantes menos avanzados justifiquen disponer de la imagen, sobre todo, por razones técnicas y los más avanzados, en cambio, por causas más profundas, deja entrever cómo ha avanzado el conocimiento y la opinión de los sujetos a lo largo de la carrera. La otra pregunta en que se abordaba este tema refuerza el papel de la imagen también para la evaluación de la TAV y sugiere que el vídeo está recomendado sobre todo para comprobar el éxito de la sincronización, a diferencia de la etapa de traducción, donde contar con el vídeo supone una ayuda sobre todo para la traslación completa del contenido del original, que no se queda en el texto, sino que emerge de la interacción imagen-texto. 
Un tercer punto sobre el que se ha preguntado a los informantes es por el sujeto evaluador de los productos audiovisuales. Las respuestas, una vez más, han servido para comprobar el mayor conocimiento de los estudiantes avanzados respecto de los iniciados, pero, además, ha constatado el rol crucial del espectador para la emisión de juicios de valor sobre los productos doblados.

Quizás el valor más importante del presente estudio sea el de demostrar que los estudiantes de traducción pueden aportar una opinión significativa sobre el concepto de calidad en TAV, y esto a pesar de que no son expertos en la materia. Más allá de que la reflexión sobre este concepto tenga un valor pedagógico per se, es evidente que constituyen un grupo interesante para la investigación, ya que se alejan del modelo de receptor pasivo que, según DíazCintas (2003: 44), es el típico en el doblaje y son capaces de emitir críticas, igual que los receptores de productos subtitulados. Esta mutación de receptor pasivo a receptor activo en un ejercicio de evaluación del doblaje es posible gracias a sus conocimientos, tanto lingüísticos como de la profesión, y esos conocimientos son también los que hacen de este grupo un perfil apropiado para evaluar el doblaje pues, según opina el propio Díaz-Cintas (2003: 70), el público a veces no tiene los conocimientos necesarios para valorar el producto.

Por otra parte, es de rigor admitir que algunas preguntas pudieron no estar bien formuladas del todo, ya que los resultados de los dos grupos de informantes fueron, a veces, atípicamente distintos; es el caso, por ejemplo, de la lengua materna. Además, vistas las escasas diferencias halladas entre los dos grupos en relación con las preguntas personales, cabría preguntarse por la inclusión de este apartado en el cuestionario. Como excepción cabe considerar la pregunta relacionada con el nivel de inglés, cuyas respuestas muestran con claridad cómo avanza el nivel de los informantes a lo largo de sus estudios. En cuanto a las preguntas relativas a la vocación, que pretendían aportar datos para responder a la primera hipótesis, es cierto que en las respuestas de los aprendices se advierte menos inseguridad, lo cual podría deberse a razones metacognitivas y a cierta madurez, alcanzada gracias, quizás, al aprendizaje desarrollado durante la carrera. Una última autocrítica respecto a la naturaleza de las preguntas es que el hecho de que haya habido que introducir en algunos casos categorías intermedias (por ejemplo, "Matiz") invita a pensar que tal vez habría sido mejor incluir preguntas cerradas. Las preguntas abiertas tienen ventajas e inconvenientes, pero un cuestionario que hubiera contado con una mezcla de preguntas de ambos tipos habría arrojado, quizás, resultados más completos; en cualquier caso, eso habría supuesto cargar de trabajo a los informantes, lo que podría haberles desmotivado para contestar. 
Los hábitos y gustos de los traductores audiovisuales en ciernes podrían estudiarse con mayor detenimiento en el futuro: llama la atención, sobre todo, su preferencia por los productos audiovisuales en versión original, lo cual hace que se alejen de las convenciones profesionales a las que, por otra parte, tendrían que acercarse si decidieran dedicarse a la traducción para el doblaje en el futuro, como muchos admitieron en el cuestionario. Este resultado inspira una nueva pregunta sobre la realidad sociológica del sector: ¿consumen en su tiempo libre los traductores audiovisuales profesionales productos doblados o en versión original? Además, y como resulta obvio, los próximos pasos llevarán a replicar el experimento en textos audiovisuales multilingües que incorporen marcas de identidad. Por ejemplo, estudiando de qué modo la sincronía determina el doblaje del multilingüismo (De Higes Andino 2014) y hasta qué punto condiciona la calidad del producto final. Son algunas de las vías que abre esta investigación, aunque la prolongación más evidente es aquella en la que se pulse la opinión acerca del concepto de calidad en el doblaje en otros sectores relacionados con la actividad, como podrían ser los profesores de traducción, los profesionales del ramo, los estudios y productoras de doblaje y los destinatarios potenciales de los productos audiovisuales traducidos.

\section{Referencias bibliográficas}

ABDAlLAH, Kristiina. (2012) Translators in production networks. Reflections on agency, quality and ethics. Joensuu: Publications of the University of Eastern Finland.

Alfaro de Carvalho, Carolina. (2012) "Quality Standards or Censorship? Language Control Policies in Cable TV Subtitles in Brazil." Meta 57:2, pp. 464-477.

ANTONINI, Rachel. (2005) "The perception of subtitled humor in Italy." Humor 18:2, pp. 209-225.

ARumí, Marta; Jordi Carrabina; Anna Matamala; Bartolomé Mesa-Lao; Pilar Orero $\&$ Javier Serrano. (2013) "Audiovisual translation learning platform: a new tool for audiovisual translation training." Hermeneus 15, pp. 39-66.

BITTNER, Hansjörg. (2011) "The Quality of Translation in Subtitling." Trans-kom 4:1, pp. 76-87.

BOURNE, Julian \& Christina Lachat. (2010) "Impacto de la norma AENOR: valoración del usuario." En: Jiménez Hurtado, Catalina; Claudia Seibel \& Ana Rodríguez Domínguez (eds.) 2010. Un corpus de cine: fundamentos teóricos y aplicados de la audiodescripción. Granada: Tragacanto, pp. 315-333.

BuCARIA, Chiara \& Delia Chiaro. (2007) "End-user perception of screen translation: the case of Italian dubbing." TradTerm 13, pp. 91-118. 
CASAS Anguita, Juana; José Ramón Repullo \& Juan de Mata Donado. (2003) "La encuesta como técnica de investigación. Elaboración de cuestionarios y tratamiento estadístico." Atención primaria 31:8, pp. 527-538.

Chaume, Frederic. (2012) Audiovisual translation: dubbing. Manchester: St. Jerome. CHAUmE, Frederic. (2007) "Quality standards in dubbing: a proposal." TradTerm 13, pp. 71-89.

Chaume, Frederic. (2004) Cine y traducción. Madrid: Cátedra.

Conde Ruano, José Tomás. (2019) Dischronies and the absence of image in the evaluation of dubbing. En: Huertas Barros, Elsa; Sonia Vandepitte \& Emilia Iglesias Fernández (eds.) 2019. Quality Assurance and Assessment Practices in Translation and Interpreting. Advances in Linguistics and Communication Studies Series. Hershey, PA: IGI Global, pp. 133-154.

DE LOS REYES, Julio. (2015) La traducción del cine para niños: un estudio sobre recepción. Castellón de la Plana: Universitat Jaume I. Tesis doctoral inédita.

Di Giovanni, Elena. (2012) "Why not ask the audience? An attempt to evaluate viewers" reception of subtitled films at festivals." En: Dalziel, Fiona; Sara Gesuato \& Maria Teresa Musacchio (eds.) 2012. A lifetime of English Studies. Essays in honour of Carol Taylor Torsello. Padua: Il Poligrafo, pp. 493-508.

DíAz CinTAS, Jorge. (2003) Teoría y práctica de la subtitulación Inglés-Español. Barcelona: Ariel.

FUENTES-LUQUE, Adrián. (2005) "La recepción de la traducción audiovisual: humor y cultura." En: Zabalbeascoa Terran, Patrick; Laura Santamaria Guinot \& Frederic Chaume Varela (eds.) 2005. La traducción audiovisual: investigación, enseñanza y profesión. Granada: Comares, pp. 139-151.

Kiraly, Don. (2000) A Social Constructivist Approach to Translator Education. Empowerment from Theory to Practice. Manchester: St. Jerome.

LÅNG, Juha; Jukka Mäkisalo; Tersia Gowases \& Sami Pietinen. (2013) "Using Eye Tracking to Study the Effect of Badly Synchronized Subtitles on the Gaze Paths of Television Viewers." New Voices in Translation Studies 10, pp. 72-86.

MAYORAL, Roberto. (2005) "Reflexiones sobre la investigación en traducción audiovisual." En: Zabalbeascoa Terran, Patrick; Laura Santamaria Guinot \& Frederic Chaume Varela (eds.) 2005. La traducción audiovisual: investigación, enseñanza y profesión. Granada: Comares, pp. 3-8.

NorD, Christiane; Masood KHOSHSALIGHEH \& Saeed AMERI. (2015) "Socio-Cultural and Technical Issues in Non-Expert Dubbing: A Case Study." International Journal of Society, Culture E Language 3:2, pp. 1-16.

Ortiz-Boix, Carla \& Anna Matamala. (2015) "Quality Assessment of Post-Edited versus Translated Wildlife Documentary Films: a Three-Level Approach.” En: O"Brien, Sharon \& Michel Simard (eds.) 2015. Proceedings of 4th Workshop on Post-Editing Technology and Practice (WPTP4). Miami, pp. 16-30. 
SEgovia, Raquel. (2005) "Semiótica textual y traducción audiovisual." En: Zabalbeascoa Terran, Patrick; Laura Santamaria Guinot \& Frederic Chaume Varela (eds.) 2005. La traducción audiovisual: investigación, enseñanza y profesión. Granada: Comares, pp. 81-86.

SOKOLI, Stavroula. (2005) "Temas de investigación en traducción audiovisual: la definición del texto audiovisual." En: Zabalbeascoa Terran, Patrick; Laura Santamaria Guinot \& Frederic Chaume Varela (eds.) 2005. La traducción audiovisual: investigación, enseñanza y profesión. Granada: Comares, pp. 177-185.

ZabAlBEASCOA, Patrick. (2008) "La credibilidad de los diálogos traducidos para audiovisuales." En: Brumme, Jenny (ed.) 2008. La oralidad fingida: descripción y traducción. Madrid: Iberoamericana, pp. 157-175.

\section{NOTA BIOGRÁFICA / BIONOTE}

JOSÉ TOMÁs CONDE RUANO es profesor de Traducción e Interpretación en la Universidad del País Vasco (UPV/EHU) y, además de colaborar con los grupos de investigación TRALIMA, PETRA y GENTT, es miembro del proyecto IDENTITRA (FFI2012-39012-C04-01T), financiado por el MINECO, que se centra en la representación de identidades en los textos audiovisuales multilingües. Se doctoró en Traducción e Interpretación por la Universidad de Granada; su tesis, sobre calidad y evaluación de traducciones, fue galardonada en 2011 con el accésit del Premio a la Mejor Tesis del Bienio, otorgado por la Asociación Ibérica de Estudios de Traducción e Interpretación. Algunos de sus últimos trabajos han aparecido en Parallèles, Cadernos de Traduçao, Trans, Hermeneus, Ibérica, Across Languages and Cultures, Meta y JosTrans. Actualmente está interesado en conocer los procesos que intervienen en la evaluación de traducciones audiovisuales.

JOSÉ TOMÁs CONDE RUANO teaches Translation and Interpreting at the University of the Basque Country (UPV/EHU) and works within the framework of several research groups, such as TRALIMA, PETRA and GENTT. Together with that, he is a member of IDENTITRA project (FFI2012-39012-C04-01T), funded by the MINECO Ministry, focused on the representation of identities of multilingual audiovisual texts. $\mathrm{PhD}$ in Translation and Interpreting from the University of Granada, his thesis deals with translation quality and evaluation and was the runner-up in the 2009-2011 Prize for the Doctoral Thesis, granted by the Iberian Association of Translation and Interpreting Studies (AIETI). Some of his last works have appeared on Parallèles, Cadernos de Traduçao, Trans, Hermeneus, Ibérica, Across Languages and Cultures, Meta and JosTrans, 
inter alia. Today, he is interested in shedding some light on the processes involved in the assessment of audiovisual translations.

\section{Anexo: Encuesta}

\section{Código:}

\begin{tabular}{|c|c|c|}
\hline \multicolumn{2}{|r|}{ PREGUNTA } & RESPUESTA \\
\hline 1 & $\begin{array}{l}\text { Para traducir productos audiovisuales, ¿crees imprescindible } \\
\text { disponer del vídeo? Justifica tu respuesta. }\end{array}$ & \\
\hline 2 & ¿Y para evaluar su calidad? Justifica tu respuesta. & \\
\hline 3 & ¿Quién debe evaluar la calidad de los doblajes? & \\
\hline 4 & ¿Es responsabilidad del traductor ajustar el texto a la imagen? & \\
\hline 5 & $\begin{array}{l}\text { Ordena de } 1 \text { (menos) a } 3 \text { (más) qué consideras más perjudicial } \\
\text { para la calidad del doblaje: } \\
\text { a) que este no coincida con el movimiento de los labios de los } \\
\text { actores; } \\
\text { b) que dure bastante más o menos que el parlamento de los } \\
\text { actores; o } \\
\text { c) que no se tenga en cuenta los movimientos corporales de los } \\
\text { actores. }\end{array}$ & \\
\hline 6 & $\begin{array}{l}\text { ¿Qué criterios hay que utilizar para evaluar la calidad de la } \\
\text { TAV? }\end{array}$ & \\
\hline 7 & ¿Sueles ver películas y series dobladas? & \\
\hline 8 & $\begin{array}{l}\text { ¿Estás familiarizado con la lectura de guiones o } \\
\text { transcripciones? }\end{array}$ & \\
\hline 9 & Indica en qué curso estás. & \\
\hline 10 & $\begin{array}{l}\text { ¿Tienes experiencia como traductor audiovisual? Justifica tu } \\
\text { respuesta. }\end{array}$ & \\
\hline 11 & ¿Tienes vocación de traductor? & \\
\hline 12 & ¿Tienes vocación de traductor audiovisual? & \\
\hline 13 & $\begin{array}{l}\text { ¿Habías visto ya alguna de las escenas que has tenido que } \\
\text { evaluar? ¿Cuáles? }\end{array}$ & \\
\hline 14 & Indica tu edad. & \\
\hline 15 & Indica tu nivel de inglés $(\mathrm{C} 2, \mathrm{C} 1, \mathrm{~B} 2, \mathrm{~B} 1, \ldots)$ & \\
\hline 16 & Indica tu lengua materna. & \\
\hline
\end{tabular}

